\title{
Why word length only matters in the left visual field
}

\author{
Carol Whitney ${ }^{\mathrm{a}, *}$, Michal Lavidor ${ }^{\mathrm{b}}$ \\ a Department of Computer Science, Neural and Cognitive Sciences Program, University of Maryland, \\ A.V. Williams Building, College Park, MD 20742, USA \\ ${ }^{\mathrm{b}}$ Department of Psychology, University of Hull, Hull, UK
}

Received 7 November 2003; received in revised form 15 April 2004; accepted 21 April 2004

\begin{abstract}
During visual word recognition, string length affects performance when stimuli are presented to the left visual field (LVF), but not when they are presented to the right visual field (RVF). Using a lexical-decision experiment, we investigated an account of this phenomenon based on the SERIOL model of letter-position encoding. Bottom-up activation patterns were adjusted via positional manipulations of letter contrast. This manipulation eliminated the LVF length effect by facilitating responses to longer words, thereby demonstrating that a length effect is not an inherent property of right-hemisphere processing. In contrast, the same manipulation slowed responses to longer words in the RVF, creating a length effect. These results show that hemisphere-specific activation patterns are the source of the asymmetry of the length effect.

(C) 2004 Elsevier Ltd. All rights reserved.

Keywords: Visual word recognition; Hemispheric asymmetry; Computational neuroscience
\end{abstract}

\section{Introduction}

It has long been known that string length has a greater impact on visual word recognition when letter strings are presented to the left visual field (LVF) than when they are presented to right visual field (RVF) (Bouma, 1973; Ellis, Young, \& Anderson, 1988; Melville, 1957; Young \& Ellis, 1985). For example, in lexical-decision experiments, RVF reaction times are unaffected by number of letters, while each additional letter increases LVF reaction times by $20-30 \mathrm{~ms}$ (Ellis et al., 1988).

\subsection{Accounts of the asymmetric length effect}

Due to the routing of optic fibers at the optic chasm, stimuli in the LVF are initially projected to the right hemisphere $(\mathrm{RH})$, while stimuli in the RVF are projected to the left hemisphere (LH). Therefore, the asymmetry of the length effect has often been taken to reflect differing modes of lexical access stemming from the LH superiority for language, with efficient, parallel processing of letters in the LH, and non-parallel processing in the RH (Bradshaw, Bradley, Gates, \& Patterson, 1977; Bub \& Lewine, 1988; Ellis et al., 1988; Young \& Ellis, 1985). However, others have argued

\footnotetext{
* Corresponding author. Tel.: +1-301-384-3391; fax: +1-301-405-6707. E-mail address: cwhitney@cs.umd.edu (C. Whitney).
}

against this interpretation, based on lack of asymmetry in serial position effects under stringent fixation control (Jordan, Patching, \& Thomas, 2003), or on the finding that a length effect emerges in the RVF at very large eccentricities (Bouma, 1973; Nazir, 2003).

Recent insights into the cortical representation of the human fovea also suggest difficulties for the dual-modes account. Mounting evidence from behavioral and TMS studies indicates that the representation of visual space is precisely split down the vertical meridian, with no bilateral representation of the fovea (Brysbaert, 1994; Brysbaert, Vitu, \& Shroyens, 1996; Lavidor \& Walsh, 2003). These studies are in line with neuroanatomical arguments against such a bilateral representation, as discussed by Leff (2004). Most damning is the fact that, given the high resolution of modern brain-imaging techniques, a bilateral representation in primary visual cortex should be detectable if it were present, yet no imaging study has found any evidence for such a representation (Leff, 2004). Thus, available evidence indicates that letters immediately to the left of fixation initially go to the RH, and letters to the right to the LH. The assumption of hemisphere-specific access modes then leads to the unlikely scenario that the two halves of a centrally fixated word are accessed by completely different mechanisms.

Imaging studies of higher brain regions involved in visual word recognition also suggest that there is a single mode of lexical access. These studies indicate that an area 
of left inferotemporal cortex provides a gateway to the word-recognition system (Cohen et al., 2000; McCandliss, Cohen, \& Dehaene, 2003; Petersen, Fox, Snyder, \& Raichle, 1990). This visual word form area (VWFA) is activated approximately $170 \mathrm{~ms}$ after presentation (Cohen et al., 2000), independently of stimulus location. While there is some debate about whether this area deserves such a moniker since it also responds to other types of stimuli and other areas also respond to letter strings (Price \& Devlin, 2003), there is strong evidence that this area is preferentially tuned to letter strings, and that brain activation becomes left-lateralized at a prelexical stage in response to letter strings (McCandliss et al., 2003; Tarkiainen, Helenius, Cornelissen, \& Salmelin, 1999).

Furthermore, masking studies suggest that information is transferred at the feature level from the RH to the $\mathrm{LH}$ during visual word recognition (Moscovitch, 1983, 1986a). Thus there seems to be a single route from visual input to the lexicon through left inferotemporal cortex.

However, a single mode of lexical access presents a quandary. How could differential effects of length arise within a single route? How can such lexical level asymmetry occur if a word is always accessed via the same mechanism?

One explanation under a single mode of lexical access is based on reading direction. Nazir $(2000,2003)$ has suggested that low-level, perceptual learning leads to the optimization of processing within the perceptual span (the area that must be visible in order for text reading to occur at normal speeds). For a language that is read from left to right, this span is approximately four characters to the left of fixation, and 14 characters to the right (Rayner, 1975), roughly corresponding to locations where there is no length effect. Therefore, Nazir, Jacobs, and O'Regan (1998) suggest that perceptual learning allows optimized processing that is independent of word length in this region. However, this account does not elucidate the underlying mechanisms. While neural bases for perceptual learning in general have been proposed (Gilbert, Sigmund, \& Crist, 2001), it is unclear what is being learned in the specific case of reading, and how this learning leads to the lack of a length effect. Thus, although it has been decades since the hemispheric asymmetry of the length effect was first discovered, the source of this robust phenomenon in the much-studied area of visual word recognition remains a mystery.

Whitney (2004) has recently addressed these questions under the SERIOL model of letter-position encoding. The SERIOL model is a theoretical framework which specifies how the early, retinotopic representation of a string is transformed into an abstract encoding of letter order (Whitney, 2001a). Aspects of these transformations differ across hemispheres, yielding asymmetric activation patterns. Such an asymmetry could potentially explain the differing effects of string length.

We have previously demonstrated that such hemispherespecific activation patterns account for a different asymmetry arising in the lexical-decision task. Under LVF/RH presen- tation, responses to words having large orthographic neighborhoods (high- $N$ words) are facilitated compared to low- $N$ words. However, this facilitation (the $N$ effect) does not occur for RVF/LH presentation (Lavidor \& Ellis, 2002a,b). Based on predictions from the SERIOL model (Whitney, 2004), we experimentally adjusted activation patterns via positional manipulations of letter contrast. The $N$ effect was negated in the LVF/RH due to speeded responses to low- $N$ words. Conversely, an $N$ effect was created in the RVF/LH because of slowed responses to low- $N$ words (Whitney \& Lavidor, 2004). Thus it was possible to modulate the $N$ effect by manipulating activation patterns, showing that such patterns are a primary locus of the $N$ effect for words.

Similar contrast manipulations should allow negation and creation of the length effect. In the experiment presented here, we test this prediction. Before describing the experiment, we first review the SERIOL model and the ensuing predictions.

\section{SERIOL model}

It is well known that neural representations increase in abstractness as distance from the periphery increases. The SERIOL model offers a theory of the representational transformations carried out by a skilled reader in the processing stream extending from primary visual cortex to lexical access. We first give an overview of the theory, and then present the levels relevant to the present experiment in more detail. For brevity, we do not include supporting experimental evidence for our assumptions; such arguments can be found elsewhere (Whitney, 2001a,b, 2002; Whitney \& Berndt, 1999).

\subsection{Overview}

The SERIOL framework is comprised of five layers: edge, feature, letter, bigram, and word. The edge layer corresponds to the earliest levels of visual processing, where the representation of the string is split across the hemispheres, and receptive fields are small. Here there is an activation pattern resulting from the acuity gradient. This gradient originates in the density of cones in the retina and is magnified into the cortex such that the amount of cortical area representing a fixed amount of visual space is highest at fixation and falls off as eccentricity increases. Thus activation level per letter (total number of neural spikes over some time period) is highest near fixation, and falls off as distance from fixation increases.

At the feature layer, units are more broadly tuned to retinal location, and the representation of the string is still split across the hemispheres. As we discuss in more detail below, the acuity gradient is converted into an activation pattern, dubbed the spatial gradient, in which activation level decreases across the string from left to right. For example, for the stimulus BIRD, B's features become the most highly 
activated, I's the next most activated, R's the next, and D's the least.

The spatial gradient then interacts with letter units that oscillate in excitability, inducing sequential firing at the letter layer. That is, B fires, then I, then R, and then D. This conversion also results in varying letter activation levels. In general, letter activations are similar to their feature activations, except that activation is increased at the final letter because its firing is not inhibited by a subsequent letter (Whitney, 2001a; Whitney \& Berndt, 1999), as is consistent with the well-known final-letter advantage. This level of representation is taken to reside in the $\mathrm{LH}$, perhaps in the region of the VWFA.

We assume that this serial representation of letter order serves separate lexical and phonological routes to the lexicon. We concentrate on the lexical route. The letters then activate bigram units which represent ordered letter pairs. That is, a bigram unit is activated any time that its constituent letters fire in the correct order. Thus, bigrams BI, IR, and $\mathrm{RD}$, as well as those corresponding to non-contiguous letter pairs (BR, BD, and ID), become activated in our example. A bigram's activation level depends on its constituent letters' activations and the time lag between the firing of those letters.

Bigrams then activate word-level representations via weighted connections. The weights on bigram-word connections record the bigram activation pattern resulting from each word. The input to a word unit is calculated in the usual way, as the dot-product of the input and weight vectors. Lateral inhibition within the word layer allows the most highly activated word unit to become the winner.

In summary, the acuity gradient at the edge layer is transformed into a monotonically decreasing spatial gradient at the feature layer. The spatial gradient is converted into serial firing at the letter layer, which is then decoded into a set of ordered letter pairs at the bigram layer. This representation contacts the word layer via weighted connections. Thus the model describes how an initial representation that is tied to retinal location is progressively transformed into a more and more abstract representation of letter order, culminating in a prelexical encoding that is based on the relationship between letters, not on their absolute position in the string (Grainger \& Whitney, 2004).

Our account of the asymmetry of the length effect focuses primarily on the creation of the spatial gradient. For the present purposes, the details of how the spatial gradient is converted into the serial encoding are not required (Interested readers may refer to Whitney, 2001a or Whitney and Berndt, 1999). Rather, the important points about the higher layers of the model are as follows. Positionally varying activations at the feature layer determine letter activations, which determine bigram activations. When a new word is learned, its corresponding bigram activation pattern is recorded on its bigram-to-word connection weights. A word-unit's activation is determined by the dot-product of its weight vector and the bigram activation vector. As a re- sult, the distribution of activation within the word level depends on how closely the bigram activation vector matches the weight vector of the target word, as we discuss in more detail below. First, however, we turn to the details of how the acuity gradient is transformed into the spatial gradient.

\subsection{Spatial gradient formation}

The proposed spatial gradient is monotonically decreasing from the first letter to the last letter, whereas the acuity gradient increases from the first letter to the fixated letter (i.e., in the LVF/RH), and decreases from the fixated letter to the last letter (i.e., in the RVF/LH). Therefore, the slope of the RH acuity gradient is in the opposite direction as required for the spatial gradient, while the slope of the LH acuity gradient is in the same direction. Thus, in the RH, the acuity gradient's slope must be inverted as features are activated. In contrast, the acuity gradient's slope can be maintained as features are activated in the LH. As a result, processing at the feature layer differs across hemispheres, with the RH performing a more extensive transformation. We assume that this hemisphere-specific processing is learned during reading acquisition, perhaps in response to a top-down attentional gradient.

First we discuss the RH processing. We propose that RH features become more highly activated by edge-level inputs than LH features. This allows the first letter's features to reach a high level of activation even if they are far from fixation. This could occur either via stronger connections from the edge to feature layers in the $\mathrm{RH}$, or via stronger self-excitation within the $\mathrm{RH}$ feature layer. We also propose that strong directional lateral inhibitory connections exist within the feature layer such that each unit inhibits units having receptive-field locations to its right. Thus, inhibitory input increases as letter-position increases, because more and more features send inhibition from the left. This strong directional inhibition overrides the slope of the acuity gradient, inverting it. So the features comprising the first letter attain a high level of activation (due to strong excitation and lack of lateral inhibition), and activation decreases towards fixation (due to sharply increasing lateral inhibition).

Note that a purely bottom-up mechanism would not suffice to transform the LVF/RH acuity gradient into the spatial gradient. This is because activation level within the spatial gradient is determined by a letter's position within the string, not by its retinal location. A bottom-up mechanism could only affect activation levels based on retinal location. In contrast, the left-to-right mechanism of lateral inhibition allows activation levels to become string-centered.

In the LH, we assume that excitatory and lateral inhibitory connections are weaker, because the acuity gradient's slope is already in the correct direction. Thus the acuity gradient is essentially maintained at the feature layer, although some directional inhibition may steepen its slope.

In addition to inhibition within hemispheres, there is also inhibition across hemispheres. The RH features inhibit the 
(a)

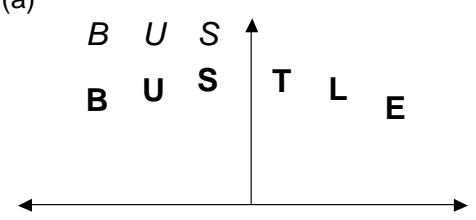

(b)

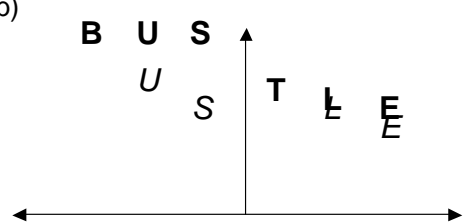

(c)

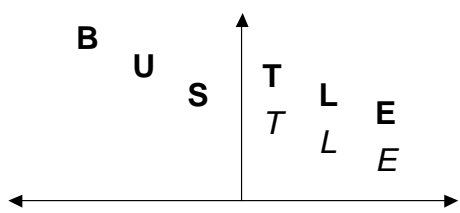

Fig. 1. Spatial gradient formation at the feature layer for a centrally fixated word (BUSTLE). The $x$-axis represents distance from fixation, while the $y$-axis represents activation level. In each panel, the boldface letters represent the activation pattern prior to the illustrated transformation, and the italics represent the result of the transformation. Panel (a) illustrates hemisphere-specific excitation. The initial activation pattern (boldface) corresponds to the acuity gradient from bottom-up input. In the LVF/RH, activation levels are boosted to a maximum level, while this is not the case for the RVF/LH (activation levels remain equivalent to bottom-up input). Panel (b) illustrates hemisphere-specific lateral inhibition, with respect to the resulting activation pattern from panel (a) (boldface). In the LVF/RH, the first letter strongly inhibits the second letter, and the first and second letters strongly inhibit the third letter to create a decreasing gradient. In the $\mathrm{RVF} / \mathrm{LH}$, lateral inhibition is less strong because the acuity gradient is consistent with the desired spatial gradient. Panel (c) illustrates cross-hemispheric inhibition, where RH letters inhibit LH letters. The resulting activation pattern (bold letters in LVF/RH and italicized letters in RVF/LH) is a monotonically decreasing gradient. For clarity, we show these transformations occurring sequentially, although they would likely occur interactively.

LH features, bringing the LH feature activations lower than those of the RH. Thus the two halves of the spatial gradient are meshed to create a strictly decreasing activation gradient from the first to the last letter (see Fig. 1).

In summary, hemisphere-specific processing is required to form the spatial gradient, due to differing acuity patterns across the visual fields. In the $\mathrm{RH}$, strong excitation and left-to-right lateral inhibition invert the acuity gradient to form the spatial gradient; in the LH, the acuity gradient serves as the spatial gradient.

\subsection{Parafoveal processing}

The hemisphere-specific transformations that are required to create the spatial gradient for a fixated word have ramifications for parafoveally presented words. Next we focus on the resulting activation patterns.

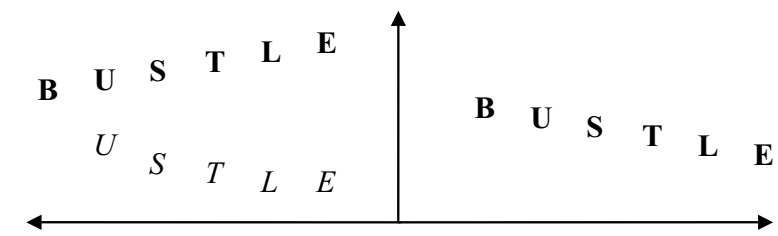

Fig. 2. Spatial gradient formation for parafoveal presentation. The boldface letters represent the activation pattern following increased bottom-up excitation. In the LVF/RH, all letters do not reach the maximal activation level, because bottom-up input levels are lower than for central fixation. Strong lateral inhibition from the first letter has a large effect on the second and third letters, because of their low levels of bottom-up input. Due the lower activations of the initial letters and the increasing activation levels of the final letters, lateral inhibition fails to create a smoothly decreasing gradient (italicized letters). In the RVF/LH, the spatial gradient remains smoothly decreasing because it is based on the acuity gradient.

Because the perceptual span in the LVF is only four letters (Rayner, 1975), acuity gradient inversion in the LVF/RH normally operates on a small number of letters of high acuity. For a long string at a large eccentricity in the LVF, these mechanisms may fail to create a smoothly decreasing spatial gradient, as follows. Strong inhibition from the first letter to the low-acuity second and third letters makes their activations quite low. However, as acuity increases for the final letters, lateral inhibition becomes insufficient, and their activations remain too high. Thus there is a sharp decrease in activation across the early string positions, and then a flattening across the final letters (see Fig. 2). In contrast, the spatial gradient remains smoothly decreasing for RVF presentation, since it is largely based on the acuity gradient. The existence of such differing activation patterns is supported by observed differential positional patterns of letter perceptability with VF (Wolford \& Hollingsworth, 1974). A computational model based on these principles closely replicated the observed patterns (Whitney, 2001a). We propose that these differing activation patterns are the source of the asymmetry of the length effect, as we discuss next.

\section{Account of the length effect and predictions}

In the SERIOL model, letter order is always encoded sequentially. First we consider how string length could fail to have an effect on reaction times despite such serial processing. For a shorter word (as compared to a longer word), the reduced amount of bottom-up input to the word layer (from fewer letters) could potentially increase the amount of time required for the network to reach response criterion. If this increased settling time for a shorter word were to exactly offset the earlier firing of its final letter, there would no effect of length. Thus we consider reaction time for central or RVF presentation to be given by:

$\mathrm{RT}($ non $-\mathrm{LVF}$, len $)=L($ len $)+S($ len $)=R$ 
where $L$ is the amount of time it takes for the final letter to start firing, and is an approximately linear function of string length, len, with slope $K$, where $K>0 . S$ is the amount of time it takes for the network to settle after the final letter starts firing, and is an approximately linear function of len with slope $-K$. Because the slopes cancel each other, their sum is a constant, $R$, giving no length effect.

Outside of this regime of exact cancellation, a length effect naturally emerges. We propose that the non-linear RH spatial gradient creates a length effect by changing the function $S$. Recall that feature-level activations are carried forward to the letter and bigram layers, and these activation levels provide the basis for the learned weights on bigram-word connections. Because the activation of a word unit is a function of the dot-product of the bigram activation vector and the weight vector, recognition will be optimal when the activation vector and the target's weight vector match (i.e., the angle between them is 0 ). In this case, the advantage of the target word-unit's activation over competitors' activations is maximized, and settling time is minimized. If the bigram activation vector diverges from its target's weight vector, activation at the word layer will be less focused on the target, and settling time will increase. This would be the case for an activation vector arising from the non-linear RH spatial gradient.

As a string length increases in the LVF, the spatial gradient becomes more and more non-linear. Thus, activation at the word layer becomes more and more diffuse, so settling time increases more and more. Therefore, we propose that settling time for LVF presentation is not a decreasing function of len, but rather is approximately constant, giving:

$\mathrm{RT}(\mathrm{LVF}$, len $)=L($ len $)+S$

This analysis implies that the length effect would disappear if a smoothly decreasing spatial gradient could be created in the LVF. Recall that for LVF parafoveal presentation, activations of the second and third letters are too low, and activations at larger positions are too high. Thus it should be possible to create a smooth gradient via an increase of bottom-up input to the second and third letters (to compensate for lateral inhibition from the first letter). Such an increase should also increase lateral inhibition to the subsequent (fourth and fifth) letters, as desired. Additionally, for words of more than five letters, a reduction of bottom-up input is probably required at the final letters in order to sufficiently compensate for their increasing acuity (i.e., to bring their activation levels low enough to make a smooth gradient) (see Fig. 3). These adjustments could be accomplished under experimental conditions by increasing contrast at the second and third positions, and reducing contrast at the sixth and higher positions.

This leads to the prediction that such a manipulation should cancel the length effect in the LVF/RH via facilitation for the longer strings. For example, for four- to six-letter strings, mean RTs to five- and six-letter strings under the above contrast manipulation should be as fast as the mean RT

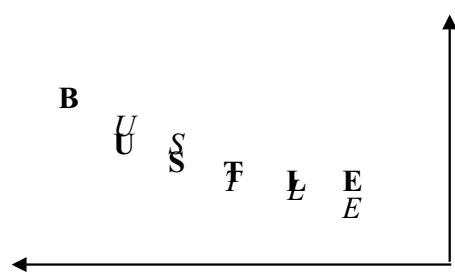

Fig. 3. Correction of the LVF/RH spatial gradient. The original gradient is given in boldface, while the corrected gradient is given in italics. Increased bottom-up input to the second and third letters raises their activation levels, and increases lateral inhibition to the fourth and fifth letters. Decreased bottom-up input to the sixth letter lowers its activation level. The result is a smoothly decreasing gradient.

to four-letter strings under normal presentation. Conversely, application of the same pattern in the RVF/LH should create a length effect due to disruption of a previously smooth spatial gradient. We tested these predictions in the following experiment.

\section{Experiment}

\subsection{Subjects}

Twenty-three right-handed, native English speakers served as subjects for a lexical-decision experiment (mean age 19.7). Ten were males, and 13 were females. All gave their informed consent to participate in the study.

\subsection{Stimuli}

Ninety-six English content words and 96 nonwords were used, with equal numbers of four-, five-, and six-letter words (32 of each). These three word sets were matched for written word frequency, orthographic neighborhood, and imageability (see Table 1). The stimuli have been published elsewhere (Lavidor \& Ellis, 2002a, Exp. 3).

Ninety-six nonwords were generated from another word pool by changing one letter, such that the nonwords were legal and pronounceable. Nonwords were also made of four, five and six letters in equal proportion.

All stimuli were presented in 14-point Helvetica lower-case font on a dark gray background of $3 \mathrm{~cd} / \mathrm{m}^{2}$. Letters were displayed at three contrast levels: high contrast

Table 1

Word frequency, orthographic neighborhood, and imageability values of word stimuli (values are taken from the MRC Psycholinguistic Database; Coltheart, 1981)

\begin{tabular}{llll}
\hline Word length & $\begin{array}{l}\text { Mean } \\
\text { frequency }\end{array}$ & $\begin{array}{l}\text { Mean } \\
\text { imageability }\end{array}$ & Mean $N$ size \\
\hline Four letters & 10.8 & 470 & 5.4 \\
Five letters & 10.5 & 510 & 5.0 \\
Six letters & 10.0 & 505 & 5.1 \\
\hline
\end{tabular}


$(c=0.64)$ white letters, medium contrast light-gray letters (high contrast reduced by 40\%), and low contrast darker-gray letters (high contrast reduced by $60 \%$ ). In the control condition, letters at all positions were presented in medium contrast. In the adjust condition, for all string lengths, the first and fourth positions were presented in medium contrast, and the second and third positions in high contrast. For five- and six-letter targets, the fifth position was presented in medium contrast. For six-letter targets, the sixth position was presented in low contrast. Thus, relative to the control condition, the second and third letters were brightened, and the sixth letter (if present) was darkened, while the other positions were presented at the same contrast level.

\subsection{Design}

Each subject was assigned to one of the two versions of the experiment. The different versions rotated the word sets across the presentation conditions (control and adjust conditions in a Latin square design). Each session began with 70 practice trials to introduce the task. Every target stimulus was presented twice, once in each visual field, giving 384 trials for each subject. Stimuli were presented in a random order with the restriction that no more than three successive words or nonwords, or three successive LVF or RVF trials occurred together. The within-subject factors were lexical status (word or nonword), length (four, five, or six letters), visual field (RVF, LVF), and presentation condition (control or adjust).

\subsection{Procedure}

Each trial began with + appearing in the center of the screen for $400 \mathrm{~ms}$, which then disappeared when the target string was presented. Targets were briefly presented for $180 \mathrm{~ms}$ at a displacement of $2.5^{\circ}$ from the fixation point to the center of the string. The subject's task was to decide, as quickly and as accurately as possible, whether the stimulus was a legal English word or a nonword.

Participants were informed that central fixation was important, and a chinrest together with a head strap were used to ensure stable head position at a distance of $50 \mathrm{~cm}$ from screen center. Participants' eye movements were monitored by an infra-red eye tracker, and were recorded for the first $700 \mathrm{~ms}$ of each trial.

\subsection{Results}

Trials in which gaze did not remain stable on the fixation cross were discarded (3\% of word trials; $5.1 \%$ of nonword trials). RTs of less than $200 \mathrm{~ms}$ and more than $1100 \mathrm{~ms}$ were also discarded either as anticipatory or excessively lengthy (discarded trials occurred infrequently, less than $3 \%$ of the total). Mean reaction times and error rates are given in Tables 2 and 3.
Table 2

Mean reaction times (and standard deviations) for word targets in $\mathrm{ms}$, and error scores as a function of target length, visual field, and presentation condition

\begin{tabular}{lcccr}
\hline & $\begin{array}{l}\text { LVF } \\
\text { control }\end{array}$ & $\begin{array}{l}\text { LVF } \\
\text { adjust }\end{array}$ & $\begin{array}{l}\text { RVF } \\
\text { control }\end{array}$ & $\begin{array}{l}\text { RVF } \\
\text { adjust }\end{array}$ \\
\hline Four letters & & & & \\
$\quad$ Mean RT & 527 & 536 & 487 & 474 \\
S.D. & 71 & 69 & 68 & 66 \\
Percent error & 15 & 18 & 12 & 7 \\
Five letters & & & & \\
$\quad$ Mean RT & 563 & 518 & 477 & 536 \\
S.D. & 70 & 67 & 70 & 72 \\
Percent error & 13 & 10 & 12 & 7 \\
Six letters & & & & \\
$\quad$ Mean RT & 594 & 535 & 490 & 548 \\
S.D. & 80 & 71 & 77 & 71 \\
Percent error & 14 & 14 & 10 & 11 \\
\hline
\end{tabular}

Repeated measures ANOVAs on reaction times (separated for words and nonwords) revealed that visual field had a significant effect $(F(1,22)=47.3, P<0.00001$ for words, ns for nonwords), with RVF words (mean RT $=502 \mathrm{~ms}$ ) responded to faster than LVF words (mean RT $=545 \mathrm{~ms}$ ). String length was significant (words: $F(2,44)=19.8, P<$ 0.001 ; nonwords: $F(2,44)=3.53, P<0.05)$, with longer latencies to longer strings. The main effect of presentation condition was not significant.

Presentation condition and visual field interacted $(F(1,22)=22.7, P<0.001$ for words; $F(1,22)=8.0$, $P<0.05$ for nonwords). This interaction was analyzed using a simple main effects analysis. For LVF stimuli, the adjust condition was faster than the control condition $(F(1,22)=6.76, P<0.05)$; For RVF stimuli, the opposite pattern was found $(F(1,22)=5.33, P<0.05)$. No interaction was found for presentation condition and length, nor for visual field and length.

The interaction between presentation condition, visual field, and word length was significant for word stimuli

Table 3

Mean reaction times (and standard deviations) for nonword targets in $\mathrm{ms}$, and error scores as a function of target length, visual field, and presentation condition

\begin{tabular}{lcccc}
\hline & $\begin{array}{l}\text { LVF } \\
\text { control }\end{array}$ & $\begin{array}{l}\text { LVF } \\
\text { adjust }\end{array}$ & $\begin{array}{l}\text { RVF } \\
\text { control }\end{array}$ & $\begin{array}{l}\text { RVF } \\
\text { adjust }\end{array}$ \\
\hline Four letters & & & & \\
$\quad$ Mean RT & 561 & 560 & 573 & 587 \\
S.D. & 88 & 82 & 82 & 89 \\
$\quad$ Percent error & 11 & 11 & 11 & 21 \\
Five letters & & & & \\
$\quad$ Mean RT & 613 & 582 & 572 & 617 \\
S.D. & 100 & 87 & 83 & 79 \\
Percent error & 13 & 11 & 10 & 14 \\
Six letters & & & & \\
$\quad$ Mean RT & 653 & 561 & 596 & 630 \\
S.D. & 88 & 93 & 84 & 89 \\
Percent error & 9 & 10 & 18 & 14 \\
\hline
\end{tabular}



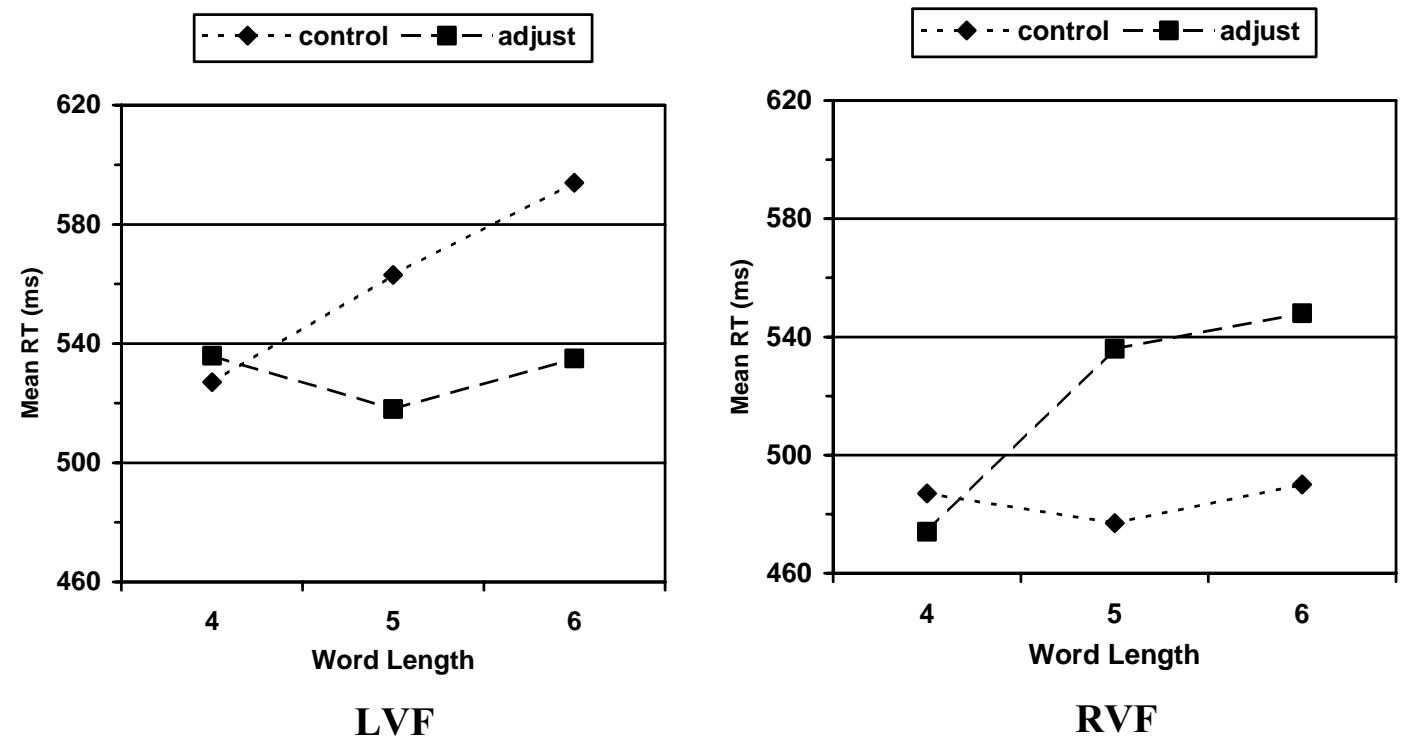

Fig. 4. Mean reaction times, by visual field, for words.

( $F(2,44)=16.84, P<0.001 ;$ ns for nonwords $)$. The triple interaction was analyzed using a simple main effects analysis. For LVF words, a length effect occurred only under the control condition $(F(2,44)=7.91, P<0.01)$. For RVF words, a length effect occurred only under the adjust condition $(F(2,44)=8.14, P<0.01)$. This pattern is clearly shown in Fig. 4. The pattern for nonwords was similar, but the three-way interaction did not reach significance.

Average error rate was $12 \%$, and no significant effects of visual field, length, or presentation condition were found.

\section{Discussion}

As predicted, the LVF/RH length effect was eliminated under the adjust condition. It cannot be argued that the effect was still present, though masked. Five- and six-letter words under the adjust condition were processed as quickly as four-letter words under the control condition, demonstrating that the length effect was completely neutralized. Thus, for the first time, a LVF reaction-time pattern that is the same as the usual RVF pattern has been achieved (i.e., one that is constant with respect to string length). This conclusively demonstrates that a length effect is not inherent feature of $\mathrm{RH}$ processing, for if it were, it would not be possible to eliminate it via a visual manipulation. Therefore, the LVF length effect does not arise from an RH-specific mode of lexical access, disproving the dual-modes theory. Since we were able to abolish the length effect via an activation-pattern correction, this indicates that the LVF activation pattern is a contributing factor to the length effect.

The appropriate contrast manipulations to neutralize the length effect were precisely predicted from the theory of spatial-gradient formation, providing strong support for this aspect of the SERIOL model. We suggest that spatial gra- dient formation provides a mechanistic account of the "perceptual learning" espoused by Nazir $(2000,2003,2004)$.

Also in line with our predictions, a length effect was created in the RVF/LH. While it may not be surprising that increased reaction times were associated with the degradation of the sixth letter in the RVF adjust condition (since it was far from fixation), we note that most of this increase was present for five-letter strings. For these strings, the only change from the control condition was positional contrast enhancement at the second and third letters. Yet, this enhancement was inhibitory in the RVF. It is unlikely that the inhibition arose solely because this enhancement reduced the visibility of nearby letters, because this manipulation had no effect on error rates or on reaction time to four-letter words, although the possibility that the low-acuity fourth letter was affected only when it was not the last letter cannot be ruled out. Nevertheless, the RVF adjust-condition results are consistent with our predictions.

The adjust condition had no effect on four-letter words, relative to their respective control conditions. However, it might be expected that RVF reaction time should increase due to a degraded spatial gradient, and LVF reaction time should decrease due to an improved spatial gradient. So why did the contrast manipulation have no effect on four-letter words? It may be the case that settling time is relatively insensitive to small differences in activation patterns for shorter words, due to the large number of competitors.

The assumption of a serial encoding at the letter layer in the SERIOL model explains why a length effect can occur. Thus, in line with the dual-modes theory, we propose that the length effect in the LVF/RH is a consequence of a serial encoding. However, we propose that the same serial encoding is used in the RVF/LH. When the spatial gradient is smooth (as in RVF/LH), the serial encoding does not manifest itself as a length effect because the amount of time it takes the 
network to settle after the final letter fires decreases with string length and cancels out the increasing time it takes for the final letter to fire. However, when the spatial gradient is not smooth (as in the LVF/RH), the settling-time advantage for longer words disappears, giving constant settling times with string length. As a result, a length effect emerges from the serial encoding. This explains how a single route of lexical access can give rise to an asymmetric length effect.

Further investigations into the length effect will involve languages read from right to left, such as Hebrew. For such languages, the spatial gradient should decrease from right to left. Thus, the consistency of the acuity gradient with respect to the spatial gradient is reversed. That is, the acuity gradient matches the spatial gradient in the LVF/RH, not the RVF/LH. This suggests that the length effect should reverse. However, experimental studies have given conflicting results. Some have shown a length effect for both visual fields (Koriat, 1985; Lavidor, Babkoff \& Faust, 2001). One has shown the predicted reversal (Nazir, Kajii, Frost \& Osaka, 2004), while another has shown the same pattern as left-to-right languages (Lavidor, Ellis \& Pansky, 2002). Overall, these results suggest that the robust asymmetry observed for left-to-right languages is not present for Hebrew, where a length effect seems to occur in both visual fields.

Based on these findings, Whitney (2004) proposed that callosal transfer to the dominant hemisphere also contributes to the length effect by preferentially degrading more lowly activated letter features. In the case of a left-to-right language, this further reduces the feature-level activations of the second and third letters. In the case of a right-to-left language, this reduces feature-level activations of the final letters, thereby delaying their firing at the letter layer, creating a length effect (i.e., the slope of the function $L$ in the RT equation is increased). Thus, it should be possible to cancel the Hebrew LVF/RH length effect by using a different experimental manipulation than in a left-to-right language-namely, by increasing bottom-up input in proportion to distance from fixation. In contrast, the same type of manipulation as in English should cancel the Hebrew RVF/LH length effect.

Thus, in left-to-right languages, callosal transfer may contribute to the non-optimality of the LVF/RH spatial gradient. This suggests that the RVF inferiority in visual word recognition for such languages arises from both inefficiency and callosal transfer (Moscovitch, 1986b). Processing is inefficient in the LVF/RH because of reading direction; the spatial gradient and the acuity gradient are incongruent. The transfer of information to the LH then exacerbates the effects of this inefficiency.

In conclusion, the present experiment has resolved the long-standing question of the source of the asymmetry of the length effect in visual word recognition. Our results demonstrate that the effect arises from hemisphere-specific activation patterns, not from hemisphere-specific modes of lexical access. This experiment was inspired by precise predictions derived from the SERIOL model. The original formulation of this model was driven by in-depth consideration of neurobiological constraints and of a wide range of complex behavioral data (Whitney, 2001a; Whitney \& Berndt, 1999). Thus, our results demonstrate the feasibility of bridging the neural and cognitive levels via the close integration of modeling and experimental work.

\section{Acknowledgements}

M.L. was supported by the BBSRC. We thank Marc Brysbaert, and the two anonymous reviewers for their helpful suggestions.

\section{References}

Bouma, H. (1973). Visual interference in the parafoveal recognition of initial and final letters of words. Vision Research, 13, 767-782.

Bradshaw, J. L., Bradley, D., Gates, A., \& Patterson, K. (1977). Serial, parallel or holistic identification of single words in two visual fields. Perception and Psychophysics, 21, 431-438.

Brysbaert, M. (1994). Interhemispheric transfer and the processing of foveally presented stimuli. Behavioral Brain Research, 64, 151-161.

Brysbaert, M., Vitu, F., \& Shroyens, W. (1996). The right visual field advantage and the optimal viewing position effect: On the relation between foveal and parafoveal word recognition. Neuropsychology, 10, 385-395.

Bub, D. N., \& Lewine, J. (1988). Different modes of word recognition in the left and right visual fields. Brain and Language, 33, 161-188.

Cohen, L., Dehaene, S., Naccache, L., Lehericy, S., Dehaene-Lambertz, G., Henaff, M. A., \& Michel, F. (2000). The visual word form area: Spatial and temporal characterization of an initial stage of reading in normal subjects and posterior split-brain patients. Brain, 125, 291-307.

Coltheart, M. (1981). The MRC psycholinguistic database. Quarterly Journal of Experimental Psychology, 33, 497-505.

Ellis, A. W., Young, A. W., \& Anderson, C. (1988). Modes of word recognition in the left and right cerebral hemispheres. Brain and Language, 35, 254-273.

Gilbert, C. D., Sigman, M., \& Crist, R. E. (2001). The neural basis of perceptual learning. Neuron, 31, 681-697.

Grainger, J., \& Whitney, C. (2004). Does the human mind read words as a whole? Trends in Cognitive Sciences, 8, 58-59.

Jordan, T. R., Patching, P. R., \& Thomas, S. M. (2003). Assessing the roles of hemispheric specialization, serial-position processing, and retinal eccentricity in lateralised word recognition. Cognitive Neuropsychology, 20, 49-71.

Koriat, A. (1985). Lateralization effects in reading pointed and unpointed Hebrew. British Journal of Psychology, 76, 161-173.

Lavidor, M., \& Ellis, A. (2002a). Word length and orthographic neighborhood size effects in the left and right cerebral hemispheres. Brain and Language, 80, 45-62.

Lavidor, M., \& Ellis, A. (2002b). Orthographic neighborhood effects in the right but not in the left cerebral hemisphere. Brain and Language, $80,63-76$.

Lavidor, M., \& Walsh, V. (2003). A magnetic stimulation examination of orthographic neighborhood effects in visual word recognition. Journal of Cognitive Neuroscience, 15, 354-363.

Lavidor, M., Babkoff, H., \& Faust, M. (2001). Analysis of standard and non-standard visual format in the two hemispheres. Neuropsychologia, 39, 430-439.

Lavidor, M., Ellis, A. W., \& Pansky, A. (2002). Case alternation and length effects in lateralized word recognition: Studies of English and Hebrew. Brain and Cognition, 50, 257-271. 
Leff, A. (2004). A historical review of the representation of the visual field in primary visual cortex with special reference to the neural mechanisms underlying macular sparing. Brain and Language, 88, 278-286.

McCandliss, B. D., Cohen, L., \& Dehaene, S. (2003). The visual word form area: Expertise for reading in the fusiform gyrus. Trends in Cognitive Science, 7, 293-299.

Melville, J. P. (1957). Word-length as a factor in differential recognition. American Journal of Psychology, 70, 316-318.

Moscovitch, M. (1983). Laterality and visual masking: Interhemispheric communication and the locus of perceptual asymmetries for words. Canadian Journal of Psychology, 37, 85-106.

Moscovitch, M. (1986a). Hemispheric specialization, interhemispheric codes and transmission times: Inferences from studies of visual masking of lateralized stimuli in normal people. In H. H. Jasper, F. LePore, \& L. M. Ptito (Eds.), Two hemispheres-One brain. New York: Alan R. Liss.

Moscovitch, M. (1986b). Afferent and efferent models of visual perceptual asymmetries: Empirical and theoretical implications. Neuropsychologia, 14, 91-114.

Nazir, T. A. (2000). Traces of print along the visual pathway. In A. Kennedy, R. Radach, D. Heller, \& J. Pynte (Eds.), Reading as a perceptual process (pp. 3-23). Amsterdam: NorthHolland.

Nazir, T. A. (2003). On hemispheric specialization and visual field effects in the perception of print: A comment on Jordan, Patching, and Thomas. Cognitive Neuropsychology, 20, 73-80.

Nazir, T. A. (2004). Reading habits, perceptual learning, and recognition of printed words. Brain and Language, 88, 294-311.

Nazir, T. A., Jacobs, A. M., \& O’Regan, J. K. (1998). Letter legibility and visual word recognition. Memory and Cognition, 26, 810821.

Nazir, T. A., Kajii, N., Frost, R., \& Osaka, N. (2004). Script characteristics modify the way we perceive isolated words: Visual field effects in the perception of French, Hebrew, Kanji and Hiragana words. In preparation

Petersen, S. E., Fox, P. T., Snyder, A. Z., \& Raichle, M. E. (1990). Activation of extrastriate and frontal cortical areas by visual words and word-like stimuli. Science, 249, 1041-1044.

Price, C. J., \& Devlin, D. T. (2003). The myth of the visual word form area. Neuroimage, 19, 473-481.

Rayner, K. (1975). Parafoveal identification during a fixation in reading. Acta Psychologia, 4, 271-282.

Tarkiainen, A., Helenius, P., Hansen, P. C., Cornelissen, P. L., \& Salmelin, R. (1999). Dynamics of letter string perception in the human occipitotemporal cortex. Brain, 122, 2119-2132.

Whitney, C. (2001a). How the brain encodes the order of letters in a printed word: The SERIOL model and selective literature review. Psychonomic Bulletin and Review, 8, 221-243.

Whitney, C. (2001b). Position-specific effects within the SERIOL framework of letter-position coding. Connection Science, 13, 235-255.

Whitney, C. (2002). An explanation of the length effect for rotated words Cognitive Systems Research, 3, 113-119.

Whitney, C. (2004). Hemisphere-specific effects in word recognition do not require hemisphere-specific modes of access. Brain and Language, 88, 279-293.

Whitney, C., \& Berndt, R. S. (1999). A new model of letter string encoding: Simulating right neglect dyslexia. Progress in Brain Research, 121, 143-163.

Whitney, C., \& Lavidor, M. (2004). Why a large orthographic neighborhood is facilitatory for presentation to the right, but not left hemisphere. Submitted for publication.

Wolford, G., \& Hollingsworth, S. (1974). Retinal location and string position as important variables in visual information processing. Perception and Psychophysics, 16, 437-442.

Young, A. W., \& Ellis, A. W. (1985). Different methods of lexical access for words presented to the left and right visual hemifields. Brain and Language, 24, 326-358. 